\title{
Modeling and simulating semantic social overlay peer-to-peer systems.
}

\begin{abstract}
The complexity of Peer-to-Peer (P2P) systems makes their analytical evaluation complicated. To conquer this problem, simulation studies are usually used to evaluate such systems. However, evolution of $\mathrm{P} 2 \mathrm{P}$ systems, from simply a Gnutella-like network to advanced overlays, makes their comparison difficult in a similar condition. Using different inputs, outputs and datasets is the main cause of this problem. On the other hand, network simulators cannot be used for such systems because of high scalability and dynamicity of P2P systems. Most network simulators can simulate few nodes in high detail without considering dynamicity of systems. In this study, a generic model, parameters and datasets are presented and used to design a flow-based $\mathrm{P} 2 \mathrm{P}$ simulator with the capability of implementing different $\mathrm{P} 2 \mathrm{P}$ protocols to simplify the evaluation of $\mathrm{P} 2 \mathrm{P}$ systems. Then, the behavior of a semantic social overlay $\mathrm{P} 2 \mathrm{P}$ system is investigated and compared with two various types of overlays, namely random and interest-based systems to show the applicability of the simulator. Although three different types of overlays have been chosen, the generic model and selected parameters used in the proposed simulator provide a uniform environment to evaluate and compare different types of overlays in similar conditions.
\end{abstract}

Keyword: Peer-to-peer computing; Social network overlay; Peer-to-peer modeling; Simulation design. 